\title{
X-ray absorption branching ratio in actinides: LDA+DMFT approach
}

\author{
J. H. Shim, K. Haule, and G. Kotliar \\ Department of Physics, Rutgers University, Piscataway, NJ 08854, USA
}

(Dated: February 28, 2022)

\begin{abstract}
To investigate the $\mathrm{x}$-ray absorption (XAS) branching ratio from the core $4 d$ to valence $5 f$ states, we set up a theoretical framework by using a combination of density functional theory in the local density approximation and Dynamical Mean Field Theory (LDA+DMFT), and apply it to several actinides. The results of the LDA+DMFT reduces to the band limit for itinerant systems and to the atomic limit for localized $f$ electrons, meaning a spectrum of $5 f$ itinerancy can be investigated. Our results provides a consistent and unified view of the XAS branching ratio for all elemental actinides, and is in good overall agreement with experiments.
\end{abstract}

PACS numbers: 78.70.Dm, 71.10..w, 71.27.+a

Understanding the physics of elemental actinide solids is an important issue for many body physics as well as for applications in nuclear power generation. In the early actinides, the $f$ electrons behave as waves delocalized through the crystal, while in the late actinides, the $f$ electrons behave as particles localized around each atom. Plutonium is near the localization-delocalization edge separating these two regimes. X-ray absortion (XAS) from the core $4 d$ to the valence $5 f$ in conjunction with atomic physics calculations has been a powerful probe of the evolution of the valence and the strength of the spin-orbit coupling across the actinide series.

A large number of spectroscopies have been applied to this problem. For example, photoemission spectroscopy of $\mathrm{Pu}$, has revealed a multiple-peak structure in the occupied part of the density of states $\$ 2.2 .3$. A combination approach of local density approximation (LDA) and Dynamical Mean Field Theory (LDA+DMFT) has allowed the interpretation of these features in terms of $f$ electrons which are delocalized at low frequencies with a mixed valent electron count $\stackrel{4}{*}$. Other interpretations of $\mathrm{Pu}$ spectroscopies using LDA+DMFT with other impurity solvers have been presented recently ${ }^{5,6,7}$.

High-energy probes such as electron energy-loss spectroscopy (EELS) or x-ray absorption spectroscopy (XAS) constitute a different set of spectroscopies which have been intensively used to study the electronic structure of the actinide series $8,9,10,11,12,13,14$. These experimental works, combined with theoretical calculations exploiting a powerful sum rule, and the electronic structure of the atom has yielded valuable insights on the degree of localization of valence $5 f$ electrons in actinides $8.9,10$ as well as the spin-orbit strength $\frac{8,10,11}{}$.

Here we address the computation of the branching ratio within DMFT. There are several motivations for this study. 1) While the atomic multiplet approach of Ref. 12 describes very successfully the majority of the data in the late actinides, it is restricted to the case that the $f$ electrons are strictly localized. It is therefore useful to extend this approach by embedding it in a more general method that captures the itinerant limit as well. 2) Many other spectroscopies of the actinides such as photoemission spectroscopies are not well described by a localized

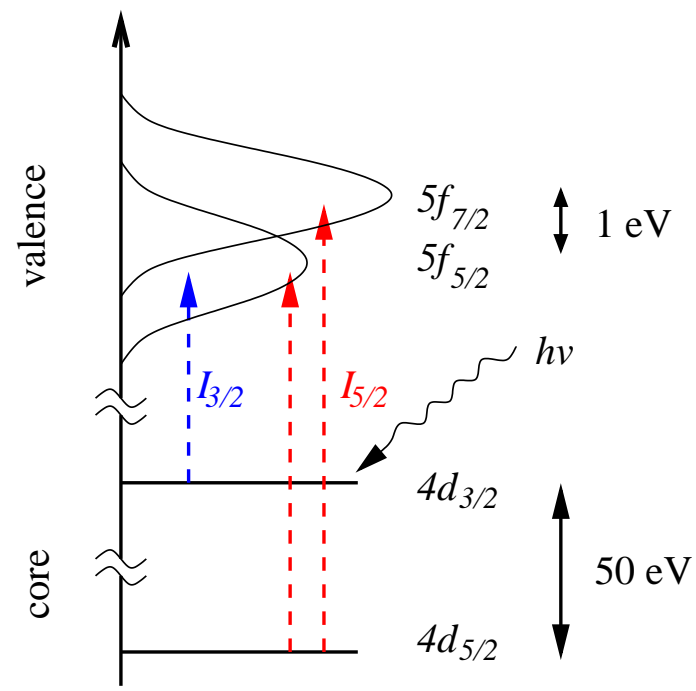

FIG. 1: Schematic diagram of the XAS from core $4 d$ to valence $5 f$ transition. The absorption intensity $I_{5 / 2}$ and $I_{3 / 2}$ correspond to $4 d_{5 / 2} \rightarrow 5 f_{5 / 2,7 / 2}$ and $4 d_{3 / 2} \rightarrow 5 f_{5 / 2}$ transitions, respectively. The core levels are clearly discretized by $4 d_{3 / 2}$ and $4 d_{5 / 2}$ level, but the valence levels are overlaped between $5 f_{5 / 2}$ and $5 f_{7 / 2}$ level due to mltiplet splittings and electron itinerancy.

model. LDA+DMFT provides a unified picture which reconciles the results of high-energy and low-energy spectroscopies. In physical terms, DMFT can describe the low-energy part of the excitations as itinerant, and the high-energy excitations as localized. Different spectroscopies weight different parts of the spectra. It is useful to incorporate the XAS branching ratio in the general LDA+DMFT formalism to achieve a unified interpretation of high energy and low energy spectroscopies. 3) The branching ratio can be expressed as a ratio of two quantities, one involving the spin-orbit coupling and the other involving the $f$ occupation. Therefore a proper theoretical interpretation of the experiments requires the evaluation of these two quantities. Until now, experimentally the occupancies were determined to be in an interval of allowed values. XAS and EELS experiments 
were used to constrain the variations of the spin-orbit coupling for that range of occupancies.

Figure 1 shows a schematic diagram of the $4 d \rightarrow$ $5 f$ transition in XAS. K. Moore and collaborators have shown that EELS experiments give equivalent information ${ }^{15,16}$. One envisions a large splitting due to spin-orbit coupling of the core levels. The electricdipole selection rule $(\Delta j=0, \pm 1)$, gives rise to two absortion lines : $I_{5 / 2}\left(4 d_{5 / 2} \rightarrow 5 f_{5 / 2,7 / 2}\right)$ and $I_{3 / 2}$ $\left(4 d_{3 / 2} \rightarrow 5 f_{5 / 2}\right)$. The branching ratio $B$ is defined by $B=I_{5 / 2} /\left(I_{5 / 2}+I_{3 / 2}\right)$.

When the spin-orbit splitting in the valence band is negligible in comparison with the different sources of broadening (due to multiplet splittings or electron itineracy) shown schematically in Fig. 1, we can neglect the spin-orbit splitting in the final states and the branching ratio $B$ is given by a statistical value of $3 / 5$, which reflects the relative degeneracies of the initial states. Hence the branching ratio is a probe of the strength of the spinorbit coupling $8,10,11$.

Detail calculations based on the isolated atomic model were shown to be consistent with the experimental result of the late actinides. However, deviations get larger when the system becomes delocalized as in U and Np. Due to the delocalization, the spin-orbit strength is apparently suppressed. We will show that although LDA is a proper approximation for describing many properties of itinerant system, it underestimates the experimental results of early actinides. So more realistic theoretical model, which can treat both the atomic and itinerant physics, is needed. The LDA+DMFT method is the most promising method for this purpose.

The spin-orbit sum-rule has been fully derived in the pioneering work of van der Laan, Thole and collaborators $12,17,18,19$, and applied to numerous systems. (for recent review, see Ref. 15.) Here we summarize the main steps of their derivation, indicating the places where the atoms are considered in a solid state environment, and where a treatment going beyond band theory and atomic multiplet physics, such as Dynamical Mean Field Theory (DMFT) is required.

The X-ray absorption resulting from a core-valence transition, is described by a term in the Hamiltonian that couples the electromagnetic field to a transition operator $T_{q}$. which in the electric dipole approximation is given by:

$$
T_{q}=\sum_{i} T_{q}(i)
$$

$T_{q}(i)$ is the atomic operator at site $i$. We consider transition from a core level denoted by $j_{c}\left(=l_{c} \pm s\right)$ to the partially occupied valence levels denoted by $j_{v}\left(=l_{v} \pm s\right)$ with absorption of $q$ polarized light.

$$
T_{q}(i)=\sum_{m_{v} m_{c}}\left\langle l_{v} s ; j_{v} m_{v}\left|\mathbf{r}_{q}\right| l_{c} s ; j_{c} m_{c}\right\rangle f_{j_{v} m_{v}}^{\dagger} d_{j_{c} m_{c}}
$$

where $d_{j_{c} m_{c}}$ is the annihilation operator of a core electron and $f_{j_{v} m_{v}}^{\dagger}$ is the creation operator of a valence electron.

The matrix element can be given as a product of a reduced matrix element containing a radial integral and an angular dependent $\operatorname{part}^{20}$.

$$
\begin{aligned}
& \left\langle l_{v} s ; j_{v} m_{v}\left|\mathbf{r}_{q}\right| l_{c} s ; j_{c} m_{c}\right\rangle=(-1)^{j_{v}-j_{c}}\left[l_{v} l_{c} j_{c}\right]^{1 / 2} \\
& \quad \times\left\{\begin{array}{lll}
j_{c} & 1 & j_{v} \\
l_{v} & s & l_{c}
\end{array}\right\}\left(\begin{array}{ccc}
j_{c} & 1 & j_{v} \\
m_{c} & q & -m_{v}
\end{array}\right)\left\langle l_{v} s\|\mathbf{r}\| l_{c} s\right\rangle,
\end{aligned}
$$

where $[a, b, \cdots]$ is shorthand for $(2 a+1)(2 b+1) \cdots$. Here, we used the Wigner-Eckart theorem. Because we are interested in the transition of given core $\left(l_{c}\right)$ and valence $\left(l_{v}\right)$ states, we will omit the reduce matrix element below.

When we consider the isotropic spectrum, where the light polarizations are averaged, the absorption intensity summed over the final states $|f\rangle$ from a many-electron ground state $|g\rangle$ is

$$
\begin{aligned}
& I=\sum_{q} \sum_{f}\left\langle g\left|T_{q}^{\dagger}\right| f\right\rangle\left\langle f\left|T_{q}\right| g\right\rangle \\
& =\sum_{q} \sum_{m_{v} m_{v}^{\prime}} \sum_{m_{c} m_{c}^{\prime}}\left\langle d_{j_{c} m_{c}^{\prime}}^{\dagger} f_{j_{v} m_{v}^{\prime}} f_{j_{v} m_{v}}^{\dagger} d_{j_{c} m_{c}}\right\rangle \\
& \times\left\langle l_{c} s ; j_{c} m_{c}^{\prime}\left|\mathbf{r}_{q}\right| l_{v} s ; j_{v} m_{v}^{\prime}\right\rangle\left\langle l_{v} s ; j_{v} m_{v}\left|\mathbf{r}_{q}\right| l_{c} s ; j_{c} m_{c}\right\rangle .
\end{aligned}
$$

Here we assume that the interference terms $\left(\left\langle T_{q}(i) T_{q}(j)\right\rangle\right.$, $i \neq j)$ are negligible which is a valid assumption when the core electron states are effectively localized. Since $|g\rangle$ does not contain holes in the core level, the core shell operators are removed by $d_{j_{c} m_{c}^{\prime}}^{\dagger}|g\rangle=0$, and the creationannihilation term in the intensity can be obtained as

$$
\left\langle d_{j_{c} m_{c}^{\prime}}^{\dagger} f_{j_{v} m_{v}^{\prime}} f_{j_{v} m_{v}}^{\dagger} d_{j_{c} m_{c}}\right\rangle=\left\langle f_{j_{v} m_{v}^{\prime}} f_{j_{v} m_{v}}^{\dagger}\right\rangle \delta_{m_{c} m_{c}^{\prime}}
$$

Where we neglected the core valence hybridization and we neglect the core-hole valence interaction, which is a reasonable approximation in $4 d \rightarrow 5 f$ transition. $\underline{\underline{8}}$

Combining Eqs. (3)-(5), the the dipole transition probability is

$$
\begin{gathered}
I=\left[l_{v} l_{c} j_{c}\right]\left\{\begin{array}{ccc}
j_{c} & 1 & j_{v} \\
l_{v} & s & l_{c}
\end{array}\right\}^{2} \sum_{m_{v} m_{v}^{\prime}}\left\langle f_{j_{v} m_{v}^{\prime}} f_{j_{v} m_{v}}^{\dagger}\right\rangle \\
\times \sum_{m_{c} q}\left(\begin{array}{ccc}
j_{c} & 1 & j_{v} \\
m_{c} & q & -m_{v}
\end{array}\right)\left(\begin{array}{ccc}
j_{c} & 1 & j_{v}^{\prime} \\
m_{c} & q & -m_{v}^{\prime}
\end{array}\right) \\
=\left[l_{v} l_{c} j_{c}\right]\left\{\begin{array}{lll}
j_{c} & 1 & j_{v} \\
l_{v} & s & l_{c}
\end{array}\right\}^{2}\left\langle n_{j_{l}}^{h}\right\rangle .
\end{gathered}
$$

We have used the normalization condition.

$$
\begin{aligned}
\sum_{m_{c} q}\left(\begin{array}{ccc}
j_{c} & 1 & j_{v} \\
m_{c} & q & -m_{v}
\end{array}\right)\left(\begin{array}{ccc}
j_{c} & 1 & j_{v}^{\prime} \\
m_{c} & q & -m_{v}^{\prime}
\end{array}\right) \\
=\left[j_{v}\right]^{-1} \delta_{j_{v} j_{v}^{\prime}} \delta_{m_{v} m_{v}^{\prime}}
\end{aligned}
$$

We consider the dipole transition from core $4 d$ states $\left(j_{c}=2 \pm 1 / 2\right)$ to valence $5 f$ states $\left(j_{v}=3 \pm 1 / 2\right)$. Using 
the relevant values of $6-j$ symbol in Eq. (5), the absorption intensity for each transition is given as

$$
\begin{gathered}
I_{3 / 2}=I\left(4 d_{3 / 2} \rightarrow 5 f_{5 / 2}\right)=\left\langle n_{5 / 2}^{h}\right\rangle\left(2 l_{v}+1\right)\left(l_{v}-1\right) / l \\
I_{5 / 2}=I\left(4 d_{5 / 2} \rightarrow 5 f_{5 / 2}\right)+I\left(4 d_{5 / 2} \rightarrow 5 f_{7 / 2}\right) \\
=\left\langle n_{5 / 2}^{h}\right\rangle / l_{v}+\left\langle n_{7 / 2}^{h}\right\rangle\left(2 l_{v}-1\right) .
\end{gathered}
$$

The branching ratio for $4 d \rightarrow 5 f$ transition is given as

$$
B=\frac{I_{5 / 2}}{I_{5 / 2}+I_{3 / 2}}=\frac{\left\langle n_{7 / 2}^{h}\right\rangle+\left\langle n_{5 / 2}^{h}\right\rangle /\left[l_{v}\left(2 l_{v}-1\right)\right]}{\left\langle n_{7 / 2}^{h}\right\rangle+\left\langle n_{5 / 2}^{h}\right\rangle}
$$

For the valence states of orbital angular moment $l$, the expectation value of the angular part of the spin-orbit interaction is related to the electron occupation numbers $\left\langle n_{j_{ \pm}}\right\rangle$of the total angular momentum levels $j_{ \pm}=l \pm 1 / 2$.

$$
\begin{aligned}
\sum_{i \in f}\left\langle\mathbf{l}_{i} \cdot \mathbf{s}_{i}\right\rangle=\sum_{j=j_{ \pm}}\left\langle n_{j}\right\rangle\left[j(j+1)-l(l+1)-\frac{3}{4}\right] \\
=-(l+1)\left\langle n_{j_{-}}\right\rangle+l\left\langle n_{j_{+}}\right\rangle .
\end{aligned}
$$

Using the definitions $\left\langle n^{h}\right\rangle=\left\langle n_{5 / 2}^{h}\right\rangle+\left\langle n_{7 / 2}^{h}\right\rangle$ and Eq. (11), the spin-orbit sum rule for $4 d \rightarrow 5 f$ transition is given by

$$
B=\frac{3}{5}-\frac{4}{15} \frac{1}{\left\langle n^{h}\right\rangle} \sum_{i \in 5 f}\left\langle\mathbf{l}_{i} \cdot \mathbf{s}_{i}\right\rangle .
$$

While we have made the assumption that the core electrons are localized, this assumption is not necessary for the valence $f$ electrons. Therefore under the conditions stated before, the sum rule is valid not only in atomic system but also in solid system, and the spin-orbit strength can be estimated from the partial occupancy of valence states at a given site, theoretically. It also shows that the angular part of the spin-orbit strength is linearly related to the branching ratio in XAS and EELS12,17,18,19.

To obtain the spin-orbit strength and branching ratio, we calculated the partial occupancies $\left\langle n_{j_{ \pm}}\right\rangle$in solid system by using the LDA and LDA+DMFT method 21 . We use a relativistic version of the linearized muffintin orbital (LMTO) method for LDA calculations 22 . In LDA+DMFT method, the itinerant spd electrons are treated using LDA, and the strongly correlated $f$ electrons are considered in DMFT approach, which maps a lattice problem to a single impurity problem in a self-consistent electronic bath $\frac{21,23}{2}$. To solve the impurity problem, we used the vertex corrected onecrossing approximation ${ }^{21}$, and the results are further cross-checked by the continuous time quantum Monte Carlo method ${ }^{24}$. The Slater integrals $F^{k}(k=2,4,6)$ and spin-orbit coupling constants are computed by Cowan' atomic Hartree-Fock (HF) program with relativistic corrections ${ }^{25}$. We scale the Slater integrals by $70 \%$ to account for the screening of the solid. We take Coulomb

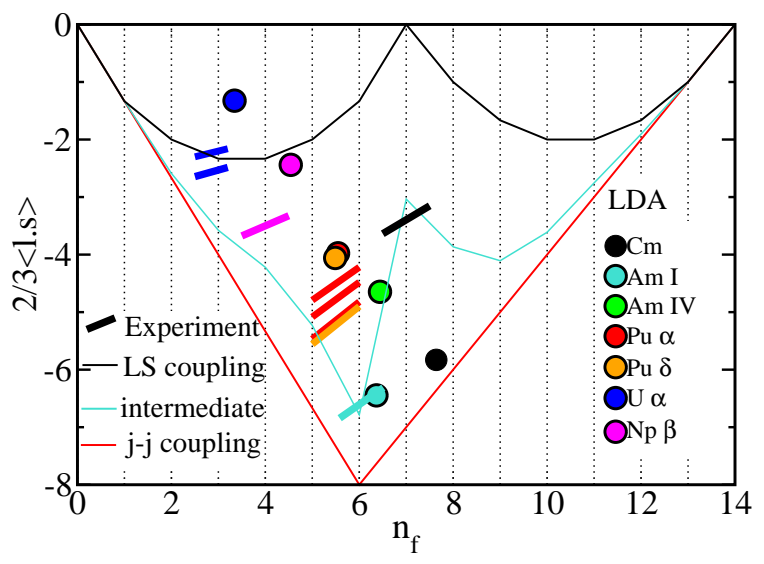

FIG. 2: The LDA expectation value of the angular part of the spin-orbit interaction. The LDA results are denoted by circles for actinide elements as a function of the $5 f$ electron count $\left(n_{f}\right)$. Paramagnetic phase is considered in all cases. Corresponding experimental data are denoted by the same color of dashes. The three common angular momentum coupling schemes are shown: $L S, j j$, and intermediate coupling schemes.

interaction $U=4.5 \mathrm{eV}$ for actinide elements and $8.0 \mathrm{eV}$ for oxides. We used an $f c c$ structure for $\mathrm{Pu}, \mathrm{Am}$, and Cm with corresponding volume of each phases, while $\alpha$ and $\beta$ - phases are used for $\mathrm{U}$ and $\mathrm{Np}$, respectively.

When the atomic interactions are turned off, the LDA+DMFT method reduces to the LDA method. Since there has not been a systematic study of how this method fares vis a vis the branching ratio, we include some LDA results in our study. In Fig. 2, we show the branching ratio of actinide elements obtained by the LDA method and compare to corresponding experimental data. We also show results for the three common angular momentum coupling schemes: $L S$ (Hund's rule is dominant), $j j$ (spin-orbit interaction is dominant), and intermediate coupling scheme (obtained from isolated atomic limit). The intermediate coupling scheme shows a good agreement with experimental results of late actinide elements, $\mathrm{Am}$ and $\mathrm{Cm}$. In Ref. 11, the calculated value of $\mathrm{Cm}$ metal overestimates the experimental value. In our result, it shows better agreement with experiment because we scaled the Slater integrals by $70 \%$, which slightly moves the intermediate coupling scheme toward $j j$ coupling scheme compared to the scaling of $80 \%$. The conventional value $80 \%$ is reasonable approximation for very localized system such as oxides. However, smaller values are necessary for very itinerant systems. For example, for transition metal elements, the scaling factor is drastically decreased for better description of spectroscopy 27 . As pointed out by Refs. 8,9 for $\alpha$-U, and Ref. 10 for $\alpha$ $\mathrm{Np}$, these metals deviate from the intermediate coupling scheme, which means these systems are delocalized and the effective spin-orbit strength decreases. 


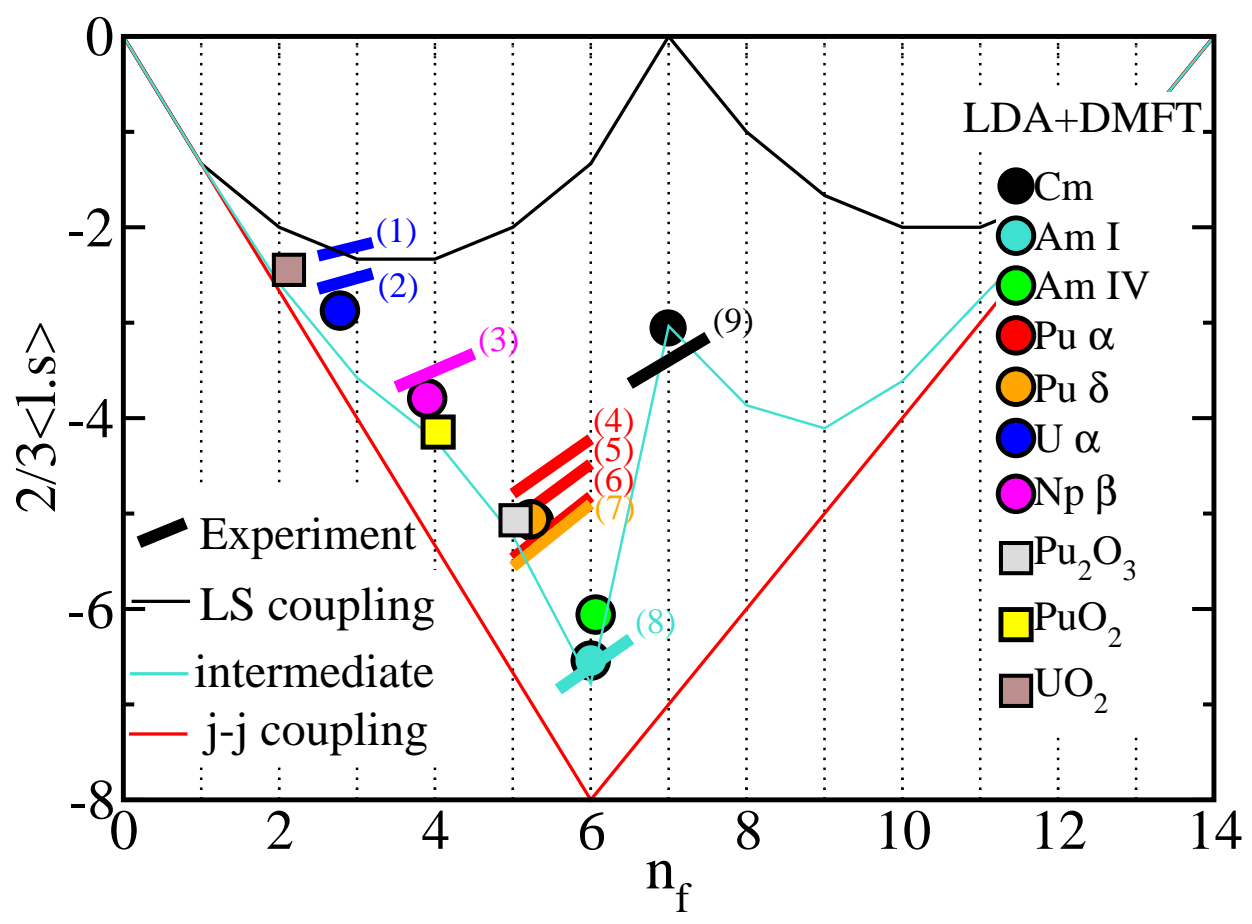

FIG. 3: The LDA+DMFT expectation value of the angular part of the spin-orbit interaction. The LDA+DMFT results are denoted by circles and rectangles for actinide elements and oxides, respectively. The experimental results obtained from EELS and XAS are denoted by thick dashes(1-8), because the number of electrons are not defined in the experiments. (1) $\alpha$-U XAS ${ }^{26}$, (2) $\alpha$-U EELS ${ }^{8}{ }^{13},(3) \alpha$-Np XAS ${ }^{10}$, (4) $\alpha$-Pu XAS ${ }^{8}$, (5) $\alpha$-Pu EELS ${ }^{8}$, (6) $\alpha$-Pu EELS ${ }^{13}$, (7) $\delta$-Pu EELS ${ }^{13}$, (8) Am I EELS ${ }^{11}$, (9) Cm EELS ${ }^{11}$.

The LDA results are in overall disagreement with experimental data and the intermediate coupling scheme. From $\mathrm{U}$ to $\mathrm{Pu}$ the spin-orbit strength is much underestimated. Due to the overestimated band width of the LDA method, the spin-orbit strength of the actinide element is considerably suppressed compared to the atomic cases. Only Am is well described by the LDA method due to its special configuration. There is an optimal spin-orbit stabilization of $\mathrm{Am} f^{6}$ configuration, which gives clear splitting of occupied $j=5 / 2$ and uoccupied $j=7 / 2$ states in LDA density of states. Our results show that the LDA description of the branching ratio is not proper for actinide elements, as shown in Fig. 2.

Figure 3 shows the branching ratio obtained by the LDA+DMFT calculation. Our results are in good agreement with experimental results of all actinide elements. The branching ratio of late actinides, $\mathrm{Am}$ and $\mathrm{Cm}$, are in good agreement with the intermediate coupling scheme as experimental data. In well localized system, only single atomic multiplet configuration is occupied and the system can be well approximated by an atomic problem, which is the intermediate coupling scheme. On the other hand, as the system is delocalized the $f$ electrons be- come fluctuating between various atomic configurations and exchanging electrons with the surrounding medium. As a result, the spin-orbit strength is suppressed and the branching ratio moves from the intermediate scheme towards the $L S$ coupling scheme. Note that the value for $\mathrm{U}$ and Np in Fig. B is further away from the intermediate coupling scheme than late actinides. Also note that this is not a competition between the spin-orbit iteraction and the Hund's rule coupling. This is the competition between the spin-orbit interaction and the delocalization. If the system is fully delocalized, the spin-orbit strength goes to zero and the branching ratio is just statistical value of $3 / 5$. According to our plot, $\delta$-Pu also deviates from the intermediate coupling scheme, hence this system is partly itinerant. This deviation becomes larger for earlier actinides such as $\mathrm{Np}$ and $\mathrm{U}$.

Our results for $\mathrm{U}$ and $\mathrm{Np}$ slightly overestimate the spin-orbit strength measured in experiments. Here, we neglected the interference effect between different site in the description of core-valence transition as expalined in Eq. (4). In the impurity problem, we use the one crossing approximation method, which might overestimate the localization of $f$ electrons because it is based on the local 


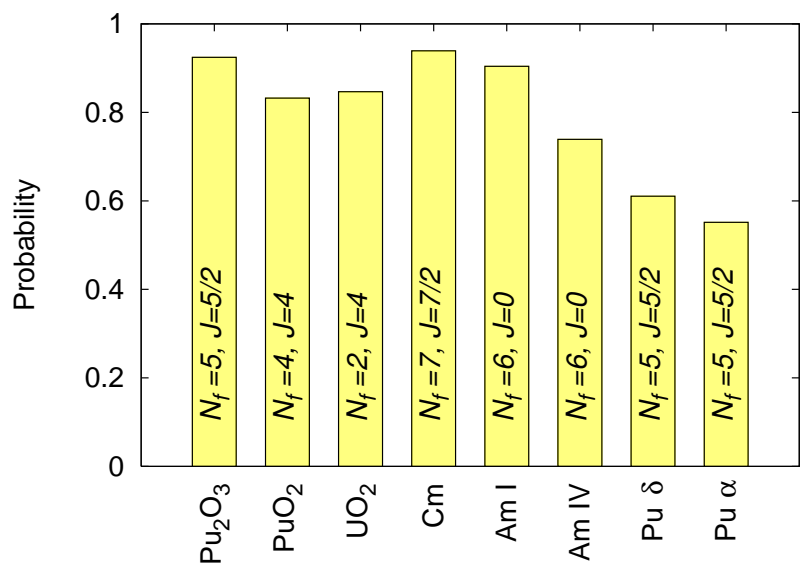

FIG. 4: Probability of the most occupied atomic ground state multiplet. The height of the peak corresponds to the fraction of the time the $f$ electrons of the solid spends in the given atomic multiplet, denoted by the $5 f$ electron count $N_{f}$ and the total spin $J$ of the atom.

atomic description. Also, the on-site Coulomb interaction $U$ should be smaller than $4.5 \mathrm{eV}$ for rather itinerant system, $\alpha-\mathrm{U}$ and $\alpha$-Np. By improving those effects, LDA+DMFT can give more delocalized branching ratio behavior.

We also investigate the change of spin-orbit strength under pressure, which are not available in atomic calculation. The volume of $\alpha-\mathrm{Pu}$ and Am IV is highly suppressed by $25 \%$ and $40 \%$ compared to the volume of $\delta$ $\mathrm{Pu}$ and Am I, respectively. The difference of $n_{f}$ and the branching ratio between $\alpha-\mathrm{Pu}$ and $\delta$-Pu is almost negligible and hard to detect, similar to EELS experiments13.15. On the other hand, there is a noticable change between Am I and Am IV. There is a transition from optimal spinorbit stabilization for $f^{6}$ to optimal exchange interaction stabilization for $f^{7}$ configuration 11 . This transition induces a significant change in branching ratio between Am I and Am IV only with slight change of $n_{f}$.
The branching ratio shows systematic deviation from atomic calculation across the actinide series. Figure 4 shows the probability of the most occupied ground state multiplet for each elements. The branching ratio of $\mathrm{Cm}$ and Am are very close to the atomic case, because the probability of the $f^{7}\left(f^{6}\right)$ ground state is $95 \%(90 \%)$ in valence histogram $\stackrel{4}{ }$, which reflects almost atomic ground states. As the atomic number is decreased, the system becomes delocalized, and the spin-orbit strength is decreased. In early actinides, the $5 f$ electrons are delocalized and they are distributed over various atomic configurations and not only in the ground state atomic multiplet. Note that $\delta$-Pu already shows deviation from localized states, as discussed in the suppressed spin-orbit strength of $\delta$-Pu. Under pressure, the $5 f$ electrons are delocalized, and the probability of atomic ground state multiplet is decreased as shown in Am IV and $\alpha-\mathrm{Pu}$.

Finally we also study actinide oxide systems $\mathrm{Pu}_{2} \mathrm{O}_{3}$, $\mathrm{PuO}_{2}$, and $\mathrm{UO}_{2}$. These materials are very important because they are used in nuclear fuels for energy generation. $\mathrm{LDA}+\mathrm{DMFT}$ predicts that these materials are localized. As shown in Fig. 3 their branching ratios are consistent with the intermediate coupling scheme. The calculated $n_{f}$ indicates that trivalent metal ion in $\mathrm{Pu}_{2} \mathrm{O}_{3}$ and tetravalent metal ion in $\mathrm{PuO}_{2}$ and $\mathrm{UO}_{2}$, which shows that these systems can be described by ionic system rather than metallic or covalent system. Recent experiments on the $4 d \rightarrow 5 f$ transition in EELS show that the branching ratio of $\mathrm{UO}_{2}$ and $\mathrm{PuO}_{2}$ are similar to that of $\alpha-\mathrm{U}$ and $\delta-\mathrm{Pu}$, respectively and it has been suggested that these actinide dioxides have covalent metal-oxide bonding, and the number of $f$ electrons can become noninteger number $\stackrel{13}{ }$. However, unpublished EELS results of the $5 d \rightarrow 5 f$ transition in $\mathrm{UO}_{2}$ and $\mathrm{PuO}_{2}$ suggest the bonding is indeed ionic with a near integer change in $5 f$ occupancy ${ }^{28}$. So, further experimental work is required to investigate why the $5 d$ and $4 d$ spectra show ionic and covalent bonding nature, respectively.

Acknowledgment: We acknowledge useful discussions with K. T. Moore.
1 A. J. Arko et al., Phys. Rev. B 62, 1773 (2000).

2 J. G. Tobin et al., Phys. Rev. B 68, 155109 (2003).

3 T. Gouder et al., Phys. Rev. B 71, 165101 (2005).

4 J. H. Shim, K. Haule, and G. Kotliar, Nature 446, 513 (2007).

5 A. Shick et al., Europhys. Lett. 77, 17003 (2007).

6 J. X. Zhu et al., Phys. Rev. B 76, 245118 (2007).

7 C. A. Marianetti, K. Haule, G. Kotliar, and M. J. Fluss, Phys. Rev. Lett. 101, 056403 (2008).

8 G. van der Laan et al., Phys. Rev. Lett. 93, 097401 (2004).

9 J. G. Tobin et al., Phys. Rev. B 72, 085109 (2005).

10 K. T. Moore, G. van der Laan, M. A. Wall, A.J. Schwartz, and R. G. Haire. Phys. Rev. B 76, 073105 (2007).

11 K. T. Moore et al. Phys. Rev. Lett. 98, 236402 (2007).

12 G. van der Laan and B. T. Thole, Phys. Rev. B 53, 14458
(1996).

13 K. T. Moore et al. Phys. Rev. B 73, 033109 (2006).

14 M. T. Butterfield, K. T. Moore, G. van der Laan, M. A. Wall, and R. G. Haire, Phys. Rev. B 77, 113109 (2008).

15 K. T. Moore and G. vand der Laan, arXiv:0807.0416 (2008)

16 K. T. Moore et al. Philos. Mag. 84, 1039 (2004).

17 B. T. Thole and G. van der Laan, Phys. Rev. B 38, 1358 (1988).

18 G. van der Laan and B. T. Thole, Phys. Rev. Lett. 60, 1977 (1988).

19 B. T. Thole and G. van der Laan, Phys. Rev. A 38, 1943 (1988).

20 G. van der Laan, Phys. Rev. B 57, 112 (1998).

21 G. Kotliar et al., Rev. Mod. Phys. 78, 865 (2006).

22 S. Y. Savrasov, Phys. Rev. B 54, 16470 (1996). 
23 A. Georges, G. Kotliar, W. Krauth, and M. J. Rozenberg, Rev. Mod. Phys. 68, 13 (1996).

${ }^{24}$ K. Haule, Phys. Rev. B 75, 155113 (2007).

25 R. D. Cowan, The Theory of Atomic Structure and Spectra (Univ. California Press, Berkeley, 1981).
${ }^{26}$ G. Kalkowski, G. Kaindl, W. D. Brewer, and W. Krone, Phys. Rev. B 35, 2667 (1987).

27 G. van der Laan, Phys. Rev. B 51, 240 (1995).

${ }^{28}$ K. T. Moore, (personal communication). 